\title{
Review found little difference between sandblasted and acid-etched (SLA) dental implants and modified surface (SLActive) implants
}

\begin{abstract}
ed from
Chambrone L, Shibli JA, Mercúrio CE, Cardoso B, Preshaw PM. Clin Oral Implants Res 2014; doi: 10.1111/clr.12347

Address for correspondence: Leandro Chambrone, Rua Antonio Pinto Guedes,

\section{Question: What are the survival rates of sandblasted and acid-etched (SLA) dental implants and modified surface (SLActive) implants submitted to immediate or early occlusal loading?}

Efficacy of standard (SLA) and modified sandblasted and acid-etched (SLActive) dental implants in promoting immediate and/or early occlusal loading protocols: a systematic review of prospective studies. 626 Mogi das Cruzes, SP 08820-430, Brazil. E-mail: leandro_chambrone@hotmail.com

Data sources Medline, Embase, the Cochrane Oral Health Group's Trials Register and OpenGREY databases were searched together with the reference lists of identified articles.

Study selection Randomised controlled trials (RCTs) and prospective cohort studies of at least six month duration were included. Studies/ case series in which there was only one implant surface (SLA or SLActive) and one loading protocol (immediate or early) were also considered.

Data extraction and synthesis Two reviewers screened, selected and abstracted data, independently. RCTs were assessed for quality using the Cochrane risk of bias approach and observational studies using the Newcastle-Ottawa scale (NOS). The primary outcomes were changes from baseline to follow-up of clinical attachment level (CAL), probing depth (PD) and radiographic changes in the peri-implant bone level and number of implants lost. Cumulative survival rates (\%) of each included study were calculated.

Results Twenty-three articles reporting 19 studies (seven RCTs; 12 prospective observational studies) were included. The seven RCTs included 407 patients with 853 implants (8\% titanium plasmasprayed, $41.5 \%$ SLA and $50.5 \%$ SLActive). Only one RCT was considered to be at low risk of bias, the others were considered to be at unclear risk. The 12 observational studies included 1394 SLA and 145 SLActive implants and were considered to be of medium methodological quality based on the NOS. A narrative summary of the studies was undertaken owing to marked heterogeneity of the loading periods, types of implants described and lack of occurrence of the outcome of interest. There were no significant differences reported in the studies in relation to implant loss or clinical parameters between the immediate/early loading and delayed loading protocols. Overall, $95 \%$ of SLA and $97 \%$ of SLActive implants still survive at the end of follow-up.

Conclusions Despite the positive findings achieved by the included studies, few RCTs were available for analysis for SLActive implants. Study heterogeneity, scarcity of data and the lack of pooled estimates represent a limitation between studies' comparisons and should be considered when interpreting the present findings.

\section{Commentary}

At the molecular level, surface topography and chemical composition have been shown to play a critical role in the predictability of the implant-to-bone response and therefore, the successful osseointegration of a dental implant. ${ }^{1}$ While numerous studies have reported on the various effects that surface coatings and chemistry have on the early stages of bone healing, there has been a reported need to evaluate whether or not surface topography and chemistry measurably influence the clinical outcome, especially in terms of loading times. This systematic review chose to test the hypothesis that SLA and/or SLActive (Straumann AG, Basel, Switzerland) surfaces can safely decrease the period of time necessary for osseointegration.

As reported, both the SLA (Sandblasted with Long grit corundum followed by Acid etching with Sulphuric and Hydrochloric acid) surface, introduced in 1997, and the SLActive surface, introduced in 2005, have a strong track record of clinical success. Both SLA and SLActive surfaces are made of cold worked titanium (grade 2) and are produced with the same sandblasting and acid-etching technique, but they differ in that the SLActive implants are rinsed under nitrogen protection to prevent exposure to air and are then stored in a sealed glass tube containing isotonic $\mathrm{NaCl}$ solution as opposed to dry storage. As described by Rupp et al., ${ }^{2}$ this contamination-reducing storage method allows the SLActive implant to have a higher surface energy and be more hydrophilic in nature than the SLA implant. Higher surface energy and hydrophilicity are important surface characteristics that facilitate a stronger cell reaction and bone tissue response in the early phase of bone healing. ${ }^{3}$

In order to test their hypothesis, the authors had to evaluate the efficacy of SLA and SLActive implants when using an immediate or early loading protocol. Loading protocols continue to be a focus of research interest and as such, continue to generate relevant clinical data as new studies emerge. While not definitive, the latest Cochrane Database Systematic Review evaluating different times for loading dental implants, ${ }^{4}$ showed that there was no convincing evidence of a clinically important difference in prosthesis failure, implant failure or bone loss associated with different loading times of dental implants. The conclusion has also been drawn that should the patient wish to shorten their treatment time and should the practitioner deem immediate loading to be appropriate for their patient's specific situation, this option would be an acceptable alternative to conventional loading protocols. ${ }^{5}$ 
The authors employed a sound methodology in their study and performed a detailed review of the available literature which included reviewing papers published in all languages. Following an analysis of the 447 potentially eligible articles identified in their search, seven RCTs and 12 prospective observational studies were chosen for inclusion, which when combined, accounted for 946 subjects and 2464 implants. The authors had well-defined primary and secondary outcome measures, however it has been reported that ideal dental implant outcome measurements should also capture aspects directly related to the treatment goal of patient wellbeing. Thus outcome measurements related to implant-supported rehabilitation should not be limited to implant survival or success rates, but when appropriate should also include the functional performance and aesthetic aspects of the entire rehabilitation as well as the health status of the peri-implant tissues. Ideally, any assessment should also include patient-reported outcomes. ${ }^{6}$

To determine the risk of bias for the seven RCTs, the authors utilised the Cochrane Handbook for Systematic reviews of Interventions, ${ }^{7}$ and categorised the included studies accordingly. They also noted that their inability to perform pooled estimates (pairwise metaanalyses) would be considered to be a limitation of the study. This inherent study heterogeneity led them to use a narrative synthesis, where a subjective rather than statistical, methodology is used. Their narrative synthesis was in accordance with the Cochrane Handbook, but this can be considered a second best approach due to the fact that any statistical data could be manipulated. As the Cochrane Handbook argues, 'there is a possibility that systematic reviews adopting a narrative approach to synthesis will be prone to bias, and may generate unsound conclusions leading to harmful decisions'. It is apparent that the authors understand this limitation and to their credit, explain that this process should be considered when interpreting their findings. When discussing potential bias, it is also important to acknowledge that four of the seven RCTs included in this systematic review were supported by Straumann AG, the manufacturer of the SLA and SLActive dental implants being investigated.

Observational studies are always at a greater risk of bias and the effects of confounding than well designed RCTs, and in order to address this concern the authors chose to use the NewcastleOttawa scale to evaluate the methodological quality of the included publications. Although this risk of bias assessment tool for observational studies is recommended by the Cochrane

\section{Practice points}

- The reported clinical differences between the survival rates of the two types of surface topography and chemical composition analysed were very small (95\% SLA and 97\% SLActive survival rate).

- The results of immediate (48 hours or less) or early ( $>48$ hours and $<3$ months) occlusal loading protocols in this systematic review were comparable to reports in the literature of those using a delayed loading protocol (three-six months).

Collaboration and it is simpler to use than other tools for assessing methodological quality or risk of bias, it should be mentioned that it is not without its detractors.

The article was clear in stating that since the SLActive implant was only relatively recently introduced (2005), there were few RCTs available for inclusion in the study, which resulted in a scarcity of data for analysis. This lack of longitudinal data makes it difficult to draw comparisons and derive at definitive conclusions. Once again, the authors make it clear that these issues deserve special attention as they are indicative of the limitations of this study.

Gary L. Stafford

Department of General Dental Sciences, Marquette University School of Dentistry, Milwaukee, Wisconsin USA

1. Junker R, Dimakis A, Thoneick M, Jansen JA. Effects of implant surface coatings and composition on bone integration: a systematic review. Clin Oral Implants Res 2009, 20: 185-206.

2. Rupp F, Scheideler L, Olshanska N, de Wild M, Wieland M, Geis-Gerstorfer ]. Enhancing surface free energy and hydrophilicity through chemical modification of microstructured titanium Implant surfaces. J Biomed Mater Res A 2006; 76: 323-334.

3. Wennerberg A, Albrektsson T. On implant surfaces: a review of current knowledge and opinions. Int / Oral Maxillofac Implants 2010; 25: 63-74.

4. Esposito M, Worthington HV, Coulthard P. Interventions for replacing missing teeth: different times for loading dental implants. Cochrane Database Syst Rev 2013; issue 3. [See Editors Note]

5. Stafford GL. Different loading times for dental implants - no clinically important differences? Evid Based Den. 2013; 14: 109-110.

6. Tonetti M, Palmer R, on behalf of Working Group 2 of the VII European Workshop on Periodontology. Clinical research in implant dentistry; study design, reporting and outcome measurements; consensus report of Working Group 2 of the VII European Workshop on Periodontology. / Clin Periodontol 2012; 39: Suppl 12 73-80.

7. Higgins JPT, Green S. Cochrane Handbook for Systematic Reviews of Interventions Version 5.1.0. [updated March 2011]. The Cochrane Collaboration, March 2011. Available from www.cochrane-handbook.org.

\section{Editors Note}

Since this commentary was prepared an update of the Cochrane review ${ }^{4}$ has been published which will be considered in a future issue.

Evidence-Based Dentistry (2014) 15, 87-88. doi:10.1038/sj.ebd.6401047 